\title{
Taxonomy of Nature Inspired Computational Intelligence: A Remote Sensing Perspective
}

\author{
Lavika Goel $^{1}$, Daya Gupta ${ }^{2}$,V.K. Panchal ${ }^{3}$ and Ajith Abraham ${ }^{4}$ \\ ${ }^{1,2}$ Department of Computer Engineering, Delhi Technological University (DTU), Delhi, India \\ 'goel.lavika@gmail.com,2dgupta@dce.ac.in \\ ${ }^{3}$ Defense Terrain \& Research Lab, Defense \& Research Development Organization (DRDO), Delhi, India \\ 3vkpans@ieee.org \\ ${ }^{4}$ Machine Intelligence Research Labs (MIR Labs), WA, USA \\ ${ }^{4}$ IT for Innovations, VSB - Technical University of Ostrava, Czech Republic \\ 4ajith.abraham@ieee.org
}

\begin{abstract}
The concepts in geospatial sciences are generally vague, ambiguous and imprecise. Also, a combination of spectral, spatial and radiometric resolution of space-borne sensors presents a selective and incomplete look of the geospatial feature/object under its view from the space. Recently, the nature inspired computational intelligence (CI) techniques have emerged as an efficient mechanism to handle diverse uncertainty characteristics. This paper proposes that the human-mind model based computational intelligence techniques, the artificial immune system based computational intelligence techniques; the swarm intelligence based computational intelligence techniques and the emerging geo-sciences based intelligent techniques can be considered as the four pillars of nature inspired CI techniques and hence redefines and extends the taxonomy of nature inspired CI. Researchers have shown keen interest on the applications of natural computing in divergent domains. Scanty references are available on the applications of nature inspired computing in the area of remote sensing. We hence also propose the taxonomy of the most recent nature inspired $\mathrm{CI}$ techniques that have been adapted till date for geo-spatial feature extraction and analyze their performances. We also construct a technology timeline of these recent nature inspired CI techniques.
\end{abstract}

Keywords--- Natural computation; computational intelligence; geo-spatial feature extraction.

\section{INTRODUCTION}

Multi-sensor, multi-resolution and multi-spectral geospatial information, from satellite remote sensing has become a primary \& vital source of information for critical, conclusive and instant decision making in varying real-time critical application across a wide array of domains like Environmental studies, Disaster management and Battlefield strategic planning. The Information referenced to geographic location, characteristics of natural and man-made features and boundaries on earth are termed as geospatial information. The concepts in geospatial sciences are generally vague, ambiguous and imprecise like the landuse / landcover, extent of Great Indian Desert Thar, the paleo-channels and the urban sprawl. Also, a combination of spectral, spatial and radiometric resolution of space-borne sensors presents a selective \& incomplete look of the geospatial feature/object under its view from the space. The resolution translates the satellite imageries into granular imprints of the geospatial features [22].

Recently, the nature inspired computational intelligence (CI) techniques which we comprised of techniques based on the modelization of human mind, those inspired from the concepts of artificial immune system, swarm intelligence based techniques and emerging geo-sciences based computing, have emerged as an efficient mechanism to handle diverse uncertainty characteristics. Nature inspired computing has been a crucial means of implementing machine intelligence with human-like behavior and reasoning capabilities. Researchers have shown keen interest on the applications of nature inspired computing in divergent domains. Scanty references are available on the applications of nature inspired computing in remote sensing [15]. The areas which have drawn the attention are landuse / landcover classification, battlefield assessment, obstacle free path planning, object identification, pattern classification, estimation of groundwater availability. From the above, the problem of land cover classification / feature extraction, which hitherto is looked for its solution in classical artificial intelligence platforms like Probabilistic and Bayesian, has been addressed and presented in this paper with new thoughts processing, based on nature inspired techniques of computational intelligence. Figure 1 summarizes and presents an overview of nature inspired computational intelligence and its perspective application areas along with the respective accuracy assessment metrics.

\section{NATURE INSPIRED COMPUTATIONAL INTELLIGENCE: TAXONOMY REDEFINED}

A new wide range of nature-inspired CI based algorithms has emerged from evolutionary algorithms and geo-sciences based natural phenomenon such as the 


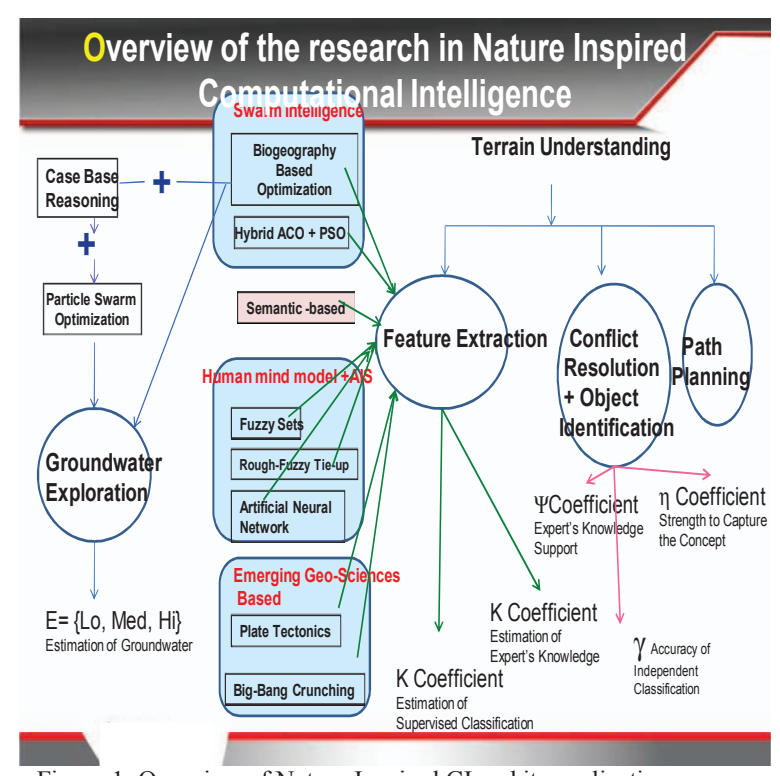

Figure 1: Overview of Nature Inspired CI and its application areas.

theories about the evolution of the earth [1], migration of species in natural habitats [2], behavior of social insects in a group [12], etc. These nature-inspired intelligent techniques can form the basis of building an optimization algorithm, which can adapt itself to suit our purpose of natural terrain feature extraction, and prove to be better by giving more accurate results than the other existing optimization techniques for certain specific applications. Also, the geo-sciences based phenomenon such as the plate tectonics, the big bang theory, etc. can be exploited to develop optimization algorithms that can be blended with the other existing evolutionary algorithms for the purpose of natural terrain feature extraction. Based on the above possibilities, figure 2 below gives the proposed taxonomy of nature inspired computational intelligence techniques that can be used independently or in an integrated way for the development of optimization algorithms that can be targeted for some specific applications. The taxonomy is described next.

\section{A. Based on the modeling of human mind}

To provide a solution to the problems posed by classical AI techniques, computational intelligence techniques based on the modelization of human mind were introduced. In effect, the role model for these techniques is the human mind. The above-mentioned computational intelligence techniques differ from the conventional (hard) computing techniques in that, unlike hard computing, they are tolerant of tolerance for imprecision, uncertainty and partial truth to achieve tractability, robustness and low solution cost. Computational models require a mathematically and logically formal representation of a problem. Computer models are used in the simulation and experimental verification of different specific and general properties of intelligence. Computational modeling can help us to understand the functional organization of a particular cognitive phenomenon. All the approaches based on the modelization of human mind tend to be generalized to the form of integrated computational models of

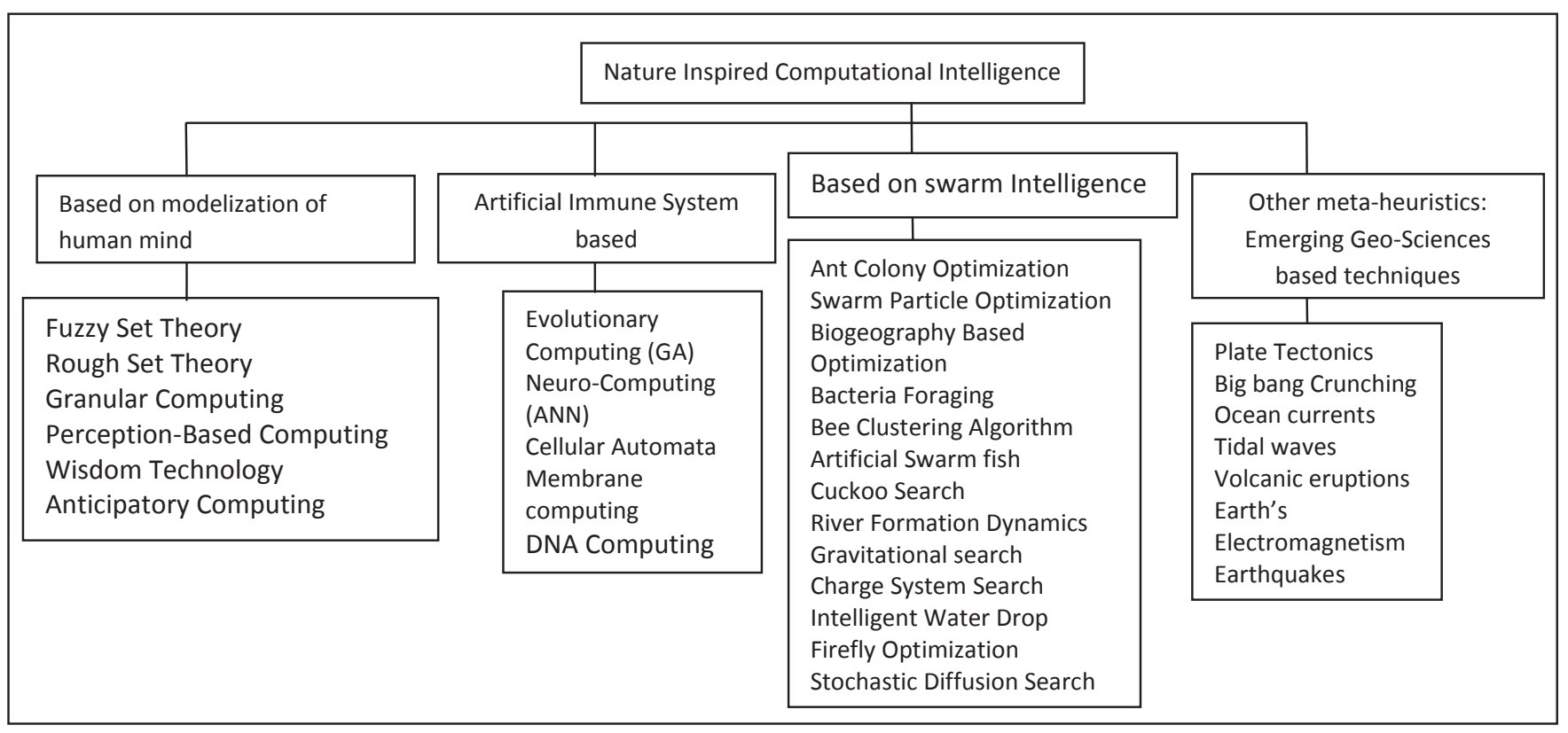

Figure 2: Proposed Taxonomy of Nature Inspired Computational Intelligence Techniques 
synthetic / abstract intelligence, in order to be applied to the explanation and improvement of individual and social / organizational decision-making and reasoning. The computational techniques proposed to be under this category are listed below.

- Fuzzy Set Theory

- Rough Set Theory

- Granular Computing

- Perception-Based Computing

- Wisdom Technology

- Anticipatory Computing

\section{B. Based on Artificial Immune System}

Artificial Immune System [3] is a framework to design a biologically inspired algorithm. It consists of the following basic elements:

- A representation for the components of the system

- A set of mechanisms to evaluate the interaction of individuals with the environment and each other. The environment is usually simulated by a set of input stimuli, one or more fitness function(s).

- Procedures of adaptation that govern the dynamics of the system i.e. how its behavior varies over time.

The computational intelligence techniques proposed to be under this category are listed below.

- Evolutionary Computing (GA)

- Neuro-computing (ANN)

- Cellular Automata

- Membrane computing

- DNA Computing

\section{Swarm Intelligence}

Swarm intelligence is any attempt to design algorithm or distributed problem-solving devices inspired by collective behavior of social insect colonies or other animal societies. This field deals with how social insects collectively perform some specific tasks in daily life (that include finding food, building or extending nest, efficiently feeding the brood, responding to external challenges, spreading alarm etc.), modeling their behavior, and using the model as a basis upon which artificial variations can be developed, either by tuning the model parameters beyond the biological relevant range or by adding non-biological features to the model. The social insect colony is a distributed system comprising of direct or indirect interactions among relatively simple (social) agents that can solve the problems in a very flexible and robust way: flexibility allow adaptation to changing environment, while robustness endows the colony with the ability to function even though some individuals may fail to perform their tasks $[1,15]$. The nature inspired techniques proposed under this category is listed below.

- Ant Colony Optimization

- Particle Swarm Optimization

- Biogeography Based Optimization

- Bacterial Foraging Optimization

- Bee Clustering Algorithm

- Artificial Swarm fish

- Cuckoo Search

- River Formation Dynamics

- Gravitational Search

- Charged System Search

- Intelligent Water Drop

- Firefly Optimization

- Stochastic Diffusion Search

\section{Emerging Geo-Sciences based Techniques}

Earth science (also known as geo-science) is an allembracing term for the sciences related to the planet Earth. It is arguably a special case in planetary science, the Earth being the only known life-bearing planet. The formal discipline of Earth sciences may include the study of the atmosphere, hydrosphere, oceans and biosphere, as well as the solid earth. Typically Earth scientists will use tools from physics, chemistry, biology, chronology and mathematics to build a quantitative understanding of how the Earth system works, and how it evolved to its current state. The following fields of science are generally categorized within the geosciences:

[1] Geology describes the rocky parts of the Earth's crust and its historic development. Major subdisciplines are mineralogy and petrology, geochemistry, geomorphology, paleontology, stratigraphy, structural geology, engineering geology and sedimentology.

[2] Physical geography covers the aspects of geomorphology, oceanography, climatology and biogeography.

[3] Geophysics and geodesy investigate the shape of the Earth, its reaction to forces and its magnetic and gravity fields. Geophysicists explore the Earth's core and mantle as well as the tectonic and seismic activity of the lithosphere.

[4] Soil science covers the outermost layer of the Earth's crust that is subject to soil formation processes. Major subdisciplines include edaphology and pedology.

[5] Oceanography and hydrology describe the marine and freshwater domains of the watery parts of the Earth. Major subdisciplines include hydrogeology 
and physical, chemical, and biological oceanography.

[6] Glaciology covers the icy parts of the Earth.

[7] Atmospheric sciences cover the gaseous parts of the Earth between the surface and the exosphere. Major subdisciplines are meteorology, climatology, atmospheric chemistry and atmospheric physics.

Plate tectonics, mountain ranges, volcanoes, and earthquakes are geological phenomena that can be explained in terms of energy transformations in the Earth's crust. Based on the above discussion, we categorize the following nature inspired techniques that can be categorized under geo-sciences as listed below:

- $\quad$ Plate Tectonics
- $\quad$ Oceanic currents
- $\quad$ Tidal waves
- $\quad$ Volcanic eruptions
- Earth's Electromagnetism
- $\quad$ Earthquakes

As seen above, several nature inspired computational intelligence techniques are available that can be adapted and implemented for real time scenarios such as in robotics, path planning, destination prediction, image classification, object identification etc. We present some of the existing nature inspired intelligent techniques that have been applied for geo-spatial feature extraction application in the next section.

\section{NATURE INSPIRED CI TECHNIQUES FOR GEO-SPATIAL FEATURE EXTRACTION}

The Indian Mission Chandrayaan-1 to the Moon in 2008 has definitely written a preface to interplanetary explorations. Also the discovery of hydroxyl water $(\mathrm{OH})$, an essential and vital geospatial feature, by Moon Impact Probe (MIP) credibly put the remote sensing at the fulcrum of geo-spatial land cover feature extraction. With the satellite remote sensing, it has become feasible to observe a vast area of the terrain and knowledge extracted out of these images. Earth Observation Satellite-based (EOS) sensors have emerged into a rapid source of multi-sensor, multi-resolution and multispectral geospatial information for decision making in varying application domains. In this study, it is attempted to explain how the nature inspired methodologies can help in working with fuzzy, granular, and ambiguous objects, under the conditions that: i. Earth objects are fuzzy in nature

ii. The inter-process collaboration is not crisp/ deterministic and times may not have exact explanation.

iii. The major source of information is the earth observation satellites. They capture different facets of the earth in different wavelength bands. Therefore, combined dataset of the images don't cover the reality in total. This implies incomplete information.

These necessitated newer procedures going and incorporating the structural knowledge into the feature extraction process. In order to encounter with the inherent uncertainty in geo-spatial data, our observation is that Nature-Inspired Computing mechanism is aptly fit into the problem-solving framework. A new wide range of nature-inspired computational algorithms is proposed which have emerged from evolutionary algorithms and geo-sciences based natural phenomenon. These natureinspired intelligent techniques can form the basis of building an optimization algorithm, which can adapt itself to suit our purpose of feature extraction and prove to be better by giving more accurate results than the other existing optimization techniques. Figure 3 provides a timeline of the most recent nature inspired CI techniques that have been adapted till date for land cover feature extraction. Table I below presents the proposed general taxonomy of the existing nature inspired computational intelligence techniques that have been used for geo-spatial feature extraction application till date.

In remote sensing the problem of feature extraction has been solved by using the traditional classical approaches of artificial intelligence like Parallel-o-piped Classification [11, 22], Minimum Distance to Mean Classification [11, 22], Maximum Likelihood Classification [11, 22] etc. A major disadvantage of the above traditional AI techniques of natural terrain feature extraction is that these techniques show limited accuracy in information retrieval and high-resolution satellite image is needed. Also these techniques are insensitive to different degrees of variance in the spectral response data. To provide a solution to the above problems, computational intelligence techniques were introduced in remote sensing for feature extraction. Computational intelligence techniques differ from the above conventional (hard) computing techniques in that, unlike hard computing, they are tolerant of imprecision, uncertainty and partial truth. The principal constituents of computational intelligence techniques categorized under the techniques inspired from modelization of human mind that have been applied for geo-spatial feature extraction are fuzzy logic [24], rough set theory [19] and Rough-Fuzzy Tie-Up [16, 18]. The main goal of the rough set analysis is to synthesize approximation of concepts from the acquired data. Fuzzy logic provides 
an inference morphology that enables approximate human reasoning capabilities to be applied to knowledge-based systems. The rough sets handles the imprecision making use of the granular knowledge structure embedded in the training / labeled dataset. The fuzzy handles the ambiguity resident in the image data set whereas, the artificial neural network is good in capturing generalization in the image data set.

Artificial Immune System is a framework to design a biologically inspired algorithm. The computational intelligence techniques proposed under this category are membrane computing [3], cellular automata, artificial neural networks and genetic algorithms. Membrane Computing deals with distributed and parallel computing models, processing multisets of symbol objects in a localized manner (evolution rules and evolving objects are encapsulated into compartments delimited by membranes), with an essential role played by the communication between compartments (and with the environment), a hierarchical arrangement of membranes, as in a cell (hence described by a tree), or a net of membranes (placed in the nodes of a graph), as in a tissue or a neural net [3]. Cellular automata concepts are built around a special class of sparse network referred to as Cellular Automata (CA). The versatility of the classification scheme is illustrated through its application in three diverse fields - data mining, image compression, and fault diagnosis. Extensive experimental results demonstrate better performance of the developed scheme over popular classification algorithms in respect of memory overhead and retrieval time with comparable classification accuracy. ANNs can be used for threedimensional terrain mapping and classification to allow the operation of mobile robots in outdoor environments using laser range finders. We make use of a multi-layer perceptron neural network to classify the terrain into navigable, partially navigable, and non-navigable. The maps generated by this approach can be used for path planning, navigation, and local obstacle avoidance. Genetic algorithms can be used for feature selection wherein feature subsets are evaluated by means of a separability index which uses covariance matrices for evaluating how spread out the probability distributions of data are in a given n-dimensional space.

The computational intelligence techniques like the fuzzy sets based classifier and path planner and the rough set classifier, which have been used recently, are not able to provide good result in case of ambiguity and also result in inaccuracy with low spatial resolution. Also these are not able to handle the continuous and the crisp data separately. Hence we shifted to the fundamentals from Swarm Intelligence, an optimized approach of terrain understanding of satellite multispectral images. Swarm Intelligence provides a good number of accuracy even with low spatial resolution image. This technique, with lower cost and higher

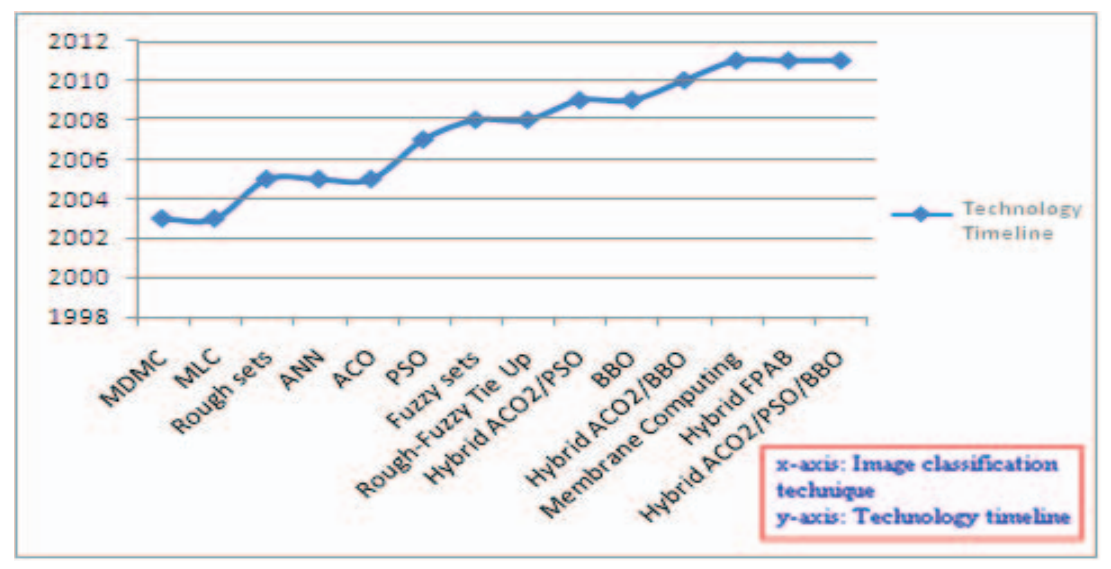

Figure 3: Technology Timeline of Nature Inspired CI Techniques in Remote Sensing 
Table I: Taxonomy of nature inspired computational intelligence techniques for feature extraction applications in remote sensing

\begin{tabular}{|c|c|c|}
\hline \multicolumn{3}{|c|}{ Nature Inspired Computational Intelligence (for Geo-spatial Feature Extraction) } \\
\hline Modelization of human mind & Artificial Immune System & Swarm Intelligence Techniques \\
\hline $\begin{array}{ll}\text { - } & \text { Rough Sets Theory } \\
\text { - } & \text { Fuzzy Sets Theory } \\
\text { - } & \text { Rough-Fuzzy Tie-Up }\end{array}$ & $\begin{array}{l}\text { - } \quad \text { Membrane Computing } \\
\text { - Artificial Neuron Network } \\
\text { (ANN) }\end{array}$ & $\begin{array}{ll}- & \text { Ant Colony Optimization (ACO) } \\
\text { - } & \text { Swarm Particle Optimization (PSO) } \\
\text { - } & \text { Hybrid ACO2/PSO Optimization } \\
\text { - } & \text { Biogeography Based Optimization (BBO) } \\
\text { - } & \text { Extended Biogeography based Optimization } \\
\text { - } & \text { Hybrid ACO2/BBO } \\
\text { - } & \text { Hybrid ACO2/PSO/BBO Optimization } \\
\text { - } & \text { Bacterial Foraging Optimization (BFO) }\end{array}$ \\
\hline
\end{tabular}

degree of accuracy, will be able to replace high resolution high cost satellite imageries. The various swarm intelligence techniques that have been adapted for the purpose of geo-spatial feature extraction till date are Ant Colony Optimization (ACO), Particle Swarm Optimization (PSO), Hybrid ACO2/PSO, Bee Colony Optimization (BCO), Bacterial Foraging Optimization (Hybrid FPAB/BBO) and Biogeography Based Optimization (BBO). ACO $[12,20]$ is used to solve discrete optimization problems. ACO can be applied to a variety of problems such as the travelling salesman problem, feature selection for dimensionality reduction, medical diagnostics, speech, and texture classification problems. However, the main limitation of ACO was the sequential selection of features, which may not lead to an optimal solution. Ant miner requires the discretization method as a pre-processing method and it is suitable only for the nominal attributes [12, 20]. Particle Swarm Optimization (PSO) $[8,23]$ is a global optimization algorithm for dealing with problems in which a best solution can be represented as a point or surface in an ndimensional space. Hybrid ACO2/PSO Optimization $[14,21]$, unlike a conventional PSO, can directly cope with the nominal attributes, without converting nominal values into numbers in a pre-processing phase. Bee Colony Optimization $(\mathrm{BCO})[4,6,7]$ is a recently proposed, nature-inspired meta-heuristic. Bee Colony Optimization is a population-based search algorithm that mimics the food foraging behavior of swarms of honey bees. It has been successfully applied to many combinatorial optimization problems, mostly in transportation, location and scheduling fields. The Algorithm for feature extraction is based on the dancing behavior of honey bees while moving from the nectar source to their hives [4]. Bacterial Foraging Optimization Algorithm [13] for feature extraction is based upon search and optimal foraging decision making capabilities of the E.Coli bacteria. The coordinates of a bacterium represent an individual solution of the optimization problem. Such a set of trial solutions converges towards the optimal solution following the foraging group dynamics of the bacteria population [13]. Biogeography Based Optimization (BBO) is an optimization technique that does not involve reproduction or the generation of "children" [2] and maintains its set of solutions from one iteration to the next, relying on migration to probabilistically adapt those solutions [2]. BBO technique has also been successfully applied for geo-spatial feature extraction [17].

\section{CONCLUSIONS}

At present, it is not possible to state which technique is best for all situations as the characteristic of each image and the circumstances for each study vary so greatly. Therefore, it is essential that each analyst understand the alternative strategies for feature extraction so that he or she may be prepared to select the most appropriate technique for the feature extraction task in hand. To this end, the concept of nature inspired computational intelligence, which can prove to be an optimized approach of feature extraction from satellite multispectral images, has been introduced in this category. These techniques with lower cost and higher degree of classification accuracy will be able to replace highresolution high cost satellite imageries.

The paper is positioned in the exploration, integration and the adaptation phases of the nature inspired computational intelligence models of problem optimization. We redefine the taxonomy of nature inspired CI techniques and since the problem in hand is the land cover feature extraction (or the satellite image classification in remote sensing terms) problem, our focus here was the development of adaptive optimization models from the terrain perspective. The Land cover feature extraction is taken as a case study. As seen 
above, extensive work has been done to develop computational intelligence inspired from nature and natural phenomenon. As far as feature extraction was concerned, a few nature inspired intelligent techniques like those of rough sets, fuzzy sets, membrane computing, ant colony optimization, particle swarm optimization, biogeography based optimization, membrane computing, bacterial foraging optimization etc. could only be adapted and implemented for real time scenarios such as in satellite imaging applications [5]. However, the nature inspired techniques of cuckoo search, firefly optimization, stochastic diffusion search, geo-sciences based techniques of plate tectonics, big bang crunching etc. have never been used to suit the land cover feature extraction applications. Certain nature inspired techniques when integrated with the existing optimization techniques can drastically improve their optimization capability hence leading to better terrain classification. We can explore these techniques further and adapt them for various applications.

\section{REFERENCES}

[1] Bonabeau, E., Dorigo, M. and Theraulaz, G., "Swarm Intelligence from Natural to Artificial System", First Edition, Oxford University Press, pp.1-24, 1999.

[2] Dan Simon, "Biogeography Based Optimization", IEEE Transactions on Evolutionary Computation, Vol. 12, No. 6, 2008.

[3] Daya Gupta, Bidisha Das, and V. K. Panchal, "A Methodical Study for the Extraction of Landscape Traits Using Membrane Computing Technique", GEM', WORLDCOMP 2011.

[4] D. Karaboga," An Idea Based On Honey Bee Swarm for Numerical Optimization, Technical Report-TR06, 2005.

[5] Demetrics Stathakis and Anthanassios Vasilakos, "Comparison of computational Intelligence based Classification Techniques for Remotely Sensed optical Image Classification", IEEE Transactions on and Remote Sensing, Vol. 44(8), 2006.

[6] Dervis Karaboga and Bahriye Akay, "A comparative study of Artificial Bee Colony algorithm", Applied Mathematics and Computation, Vol. 214, pp. 108$132,2009$.

[7] Dušan TEODOROVIĆ1,2, Mauro DELL' ORCO, "Bee Colony Optimization - A Cooperative Learning Approach To Complex Transportation Problems", Advanced OR and AI methods in Transportation, 2000 .

[8] Kennedy, J.; Eberhart, R., "Particle Swarm Optimization", Proceedings of IEEE International Conference on Neural Networks (IV), pp. 1942-1948, 1995.

[9] Lavika Goel, Daya Gupta, V.K. Panchal, "Hybrid bioinspired techniques for land cover feature extraction: A remote sensing perspective," Applied soft computing, Elsevier publications, Vol. 12(2), pp. 832849, 2011. (Impact factor 2.6)
[10] Lavika Goel, "Land cover Feature Extraction using Hybrid Swarm Intelligence Techniques-A Remote sensing perspective", ACEEE International Journal on Signal \& Image Processing, Vol. 1(3), 2010.

[11] Long, W., III; Srihann, S, Geoscience and Remote Sensing Symposium, Unsupervised and supervised classifications, "Land cover classification of SSC image: unsupervised and supervised classification using ERDAS Imagine", IGARSS '04 Proceedings, Vol. 4, pp. 20-24, 2004.

[12] M. Dorigo and T. Stuetzle, "Ant Colony Optimization", MIT Press, 2004.

[13] Navdeep Kaur Johal, Samandeep Singh and Harish Kundra, "A hybrid FPAB/BBO Algorithm for Satellite Image Classification", International Journal of Computer Applications (0975 - 8887), Vol. 6(5), 2010.

[14] N. Holden and A.A. Freitas, "A hybrid particle swarm/ant colony algorithm for the classification of hierarchical biological data", In: Proc. 2005 IEEE Swarm Intelligence Symposium (SIS-05), pp. 100-107, 2005.

[15] Omkar, S.N., Manoj, K.M., Mudigere, D. and Muley, D., "Urban Satellite Image Classification using Biologically Inspired Techniques", In Proceedings of IEEE International Symposium on Industrial Electronics, pp. 1767 - 1772, 2007.

[16] Pal, S.K. and Skowron, A., "Rough Fuzzy Hybridization: A new Trend in Decision Making", First Edition, Springer-Verlag publishers, pp.3-23, 1999.

[17] Panchal, V., Singh, P., Kaur, N., Kundra, H., "Biogeography based satellite image classification", International Journal of Computer Science and Information Security, Vol. 6 (2), pp. 269-274, 2009.

[18] Panchal VK, Singhal Naresh, Kumar Shashi, Bhakna Sonam, "Rough-Fuzzy Sets Tie-Up for Geospatial Information", Proceedings of International Conference on Emerging Scenarios in Space Technology and Applications (ESSTA2008), Vol. 1, 2008.

[19] Pawlak, Z. , "Rough set approach to knowledge-based decision support", European Journal of Operational Research, Vol. 99 (1), pp. 48-57, 1997.

[20] R.S. Parpinelli, H.S. Lopes and A.A. Freitas, "Data Mining with an Ant Colony Optimization Algorithm", IEEE Transactions on Evolutionary Computation, special issue on Ant Colony algorithms, pp. 321-332, 2002.

[21] Shelly Bansal, Daya Gupta, V.K. Panchal ,Shashi Kumar, "Remote Sensing Image Classification by Improved Swarm Inspired Techniques" in International Conference on Artificial Intelligence and Pattern Recognition (AIPR-09), 2009.

[22] Thomas M. Lillesand, Ralph W. Kiefer, "Remote Sensing and Image Interpretation", $6^{\text {th }}$ Edition, Wiley, 2008.

[23] WangDong, Wu Xiang-Bin, "Particle Swarm Intelligence Classification Algorithm for Remote Sensing Images", IEEE Pacific-Asia Workshop on Computational Intelligence and Industrial Application, 2008.

[24] Zadeh,L.A.,"Fuzzy sets", Information and Control, Vol. 8 (3), pp.338- 353, 1965. 